\title{
Individual Differences and Impact of Gender on Curvature Redirection Thresholds
}

\author{
Nguyen, Anh ; Rothacher, Yannick ; Lenggenhager, Bigna ; Brugger, Peter ; Kunz, Andreas
}

\begin{abstract}
To enable real walking in a virtual environment (VE) that is larger than the available physical space, redirection techniques that introduce multisensory conflicts between visual and nonvisual cues to manipulate different aspects of a user's trajectory could be applied. When applied within certain thresholds, these manipulations could go unnoticed and immersion remains intact. Research effort has been spent on identifying these thresholds and a wide range of thresholds was reported in different studies. These differences in thresholds could be explained by many factors such as individual differences, walking speed, or context settings such as environment design, cognitive load, distractors, etc. In this paper, we present a study to investigate the role of gender on curvature redirection thresholds (RDTs) using the maximum likelihood procedure with the classical two-alternative force choice task. Results show high variability in individuals' RDTs, and that on average women have higher curvature RDTs than men. Furthermore, results also confirm existing findings about the negative correlation between walking speed and curvature RDTs.
\end{abstract}

DOI: https://doi.org/10.1145/3225153.3225155

Posted at the Zurich Open Repository and Archive, University of Zurich

ZORA URL: https://doi.org/10.5167/uzh-162792

Book Section

Published Version

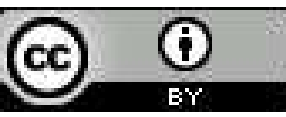

The following work is licensed under a Creative Commons: Attribution 4.0 International (CC BY 4.0) License.

Originally published at:

Nguyen, Anh; Rothacher, Yannick; Lenggenhager, Bigna; Brugger, Peter; Kunz, Andreas (2018). Individual Differences and Impact of Gender on Curvature Redirection Thresholds. In: SAP, 18. Proceedings of the 15th ACM Symposium on Applied Perception. New York, NY, USA: ACM, 5:1-5:4.

DOI: https://doi.org/10.1145/3225153.3225155 


\section{Individual Differences and Impact of Gender on Curvature Redirection Thresholds}

\author{
Anh Nguyen \\ Innovation Center Virtual Reality \\ ETH Zurich \\ nngoc@ethz.ch
}

\author{
Yannick Rothacher \\ Neuropsychology \\ University Hospital Zurich \\ yannick.rothacher@usz.ch
}

\author{
Bigna Lenggenhager \\ Cognitive Neuropsychology \\ University of Zurich \\ bigna.lenggenhager@gmail.com
}

\author{
Peter Brugger \\ Neuropsychology \\ University Hospital Zurich \\ peter.brugger@usz.ch
}

\begin{abstract}
To enable real walking in a virtual environment (VE) that is larger than the available physical space, redirection techniques that introduce multisensory conflicts between visual and nonvisual cues to manipulate different aspects of a user's trajectory could be applied. When applied within certain thresholds, these manipulations could go unnoticed and immersion remains intact. Research effort has been spent on identifying these thresholds and a wide range of thresholds was reported in different studies. These differences in thresholds could be explained by many factors such as individual differences, walking speed, or context settings such as environment design, cognitive load, distractors, etc.

In this paper, we present a study to investigate the role of gender on curvature redirection thresholds (RDTs) using the maximum likelihood procedure with the classical two-alternative force choice task. Results show high variability in individuals' RDTs, and that on average women have higher curvature RDTs than men. Furthermore, results also confirm existing findings about the negative correlation between walking speed and curvature RDTs.
\end{abstract}

\section{CCS CONCEPTS}

- Human-centered computing $\rightarrow$ Virtual reality; Empirical studies in HCI; • Applied computing $\rightarrow$ Psychology;

\section{KEYWORDS}

walking, virtual reality, multisensory conflicts, gender difference

ACM Reference Format:

Anh Nguyen, Yannick Rothacher, Bigna Lenggenhager, Peter Brugger, and Andreas Kunz. 2018. Individual Differences and Impact of Gender on Curvature Redirection Thresholds. In SAP '18: ACM Symposium on Applied Perception 2018, August 10-11, 2018, Vancouver, BC, Canada. ACM, New York, NY, USA, 4 pages. https://doi.org/10.1145/3225153.3225155

Permission to make digital or hard copies of all or part of this work for personal or classroom use is granted without fee provided that copies are not made or distributed for profit or commercial advantage and that copies bear this notice and the full citation on the first page. Copyrights for components of this work owned by others than the author(s) must be honored. Abstracting with credit is permitted. To copy otherwise, or republish, to post on servers or to redistribute to lists, requires prior specific permission and/or a fee. Request permissions from permissions@acm.org.

SAP '18, August 10-11, 2018, Vancouver, BC, Canada

(c) 2018 Copyright held by the owner/author(s). Publication rights licensed to the Association for Computing Machinery.

ACM ISBN 978-1-4503-5894-1/18/08 \&\$15.00

https://doi.org/10.1145/3225153.3225155

\author{
Andreas Kunz \\ Innovation Center Virtual Reality \\ ETH Zurich \\ kunz@iwf.mavt.ethz.ch
}

\section{INTRODUCTION}

It has been shown that the most immersive way to explore a VE is to really walk in it [Usoh et al. 1999]. However, the problem with real walking arises when the VE is much larger than the available physical space. A solution to this problem was proposed where a sensory conflict between the visual information provided through the headmounted display (HMD) and nonvisual information (vestibular and proprioceptive) is introduced [Razzaque et al. 2001]. This conflict, quantified as redirection gain, results in different virtual and real trajectories, for example: the user walks on a different path curvature in real life than in the VE (curvature redirection), the user walks slower or faster in real life than in the VE (translation redirection), and the user rotates slower or faster in real life than in the VE (rotation redirection). Nevertheless, there is a limit to this sensory conflict, beyond which it is noticeable to the user and a break in immersion occurs. Research effort has been spent on identifying this limit, so-called threshold. However, results found by different groups have quite high variance. For example, curvature thresholds found by different research groups vary considerably: $5 \mathrm{~m}$ [Grechkin et al. 2016], $10 \mathrm{~m}$ [Nitzsche et al. 2004] and $22 \mathrm{~m}$ [Steinicke et al. 2010]. An explanation for this high variance could be that these experiments had different setups and did not control for factors that affect the thresholds such as speed [Neth et al. 2011] or environment design [Hodgson et al. 2014]. However, one significant factor could be the high variance in individuals' ability to detect the sensory conflict. This ability has been proposed to correlate with one's visual dependence [Nguyen et al. 2016]. More specifically, stronger reliance on visual information could lead to higher threshold for detecting this conflict. Since a number of past research has reported gender difference in visual dependence, it could be hypothesized that there is also gender difference in the ability to detect the multisensory conflict introduced during redirected walking.

\section{RELATED WORK}

In different tasks related to spatial ability, it has been shown that women tend to have higher visual dependence than men. In a rod-and-frame test where subjects have to align a rod in a tilted frame to the gravitational vertical axis, women are more affected by the frame orientation [Tremblay et al. 2004] [Barnett-Cowan et al. 2010]. In an optokinetic drum, women perceive self-motion induced by a rotating pattern, so-called vection, faster and with stronger 
intensity [Kennedy et al. 1996] [Darlington and Smith 1998]. In the context of redirected walking, there has been research effort to investigate the effect of gender on RDTs [Bruder et al. 2009]. Even though no significant effect of gender on RDTs was found, they remarked that the number of subject was too small. Additionally, walking speed was not controlled in their experiment, which may have lead to additional noise in the result.

Given the well established gender difference in visual dependence and the limitations in terms of sample size and controlled factors of the existing study, we performed a larger scale study to investigate the effect of gender on curvature RDTs where walking speed is controlled. We also investigate if the effect of walking speed on curvature RDTs could be reproduced using a non-visual method of speed regulation. Different protocols for threshold identification are also discussed.

\section{METHODOLOGY}

\subsection{Threshold identification}

Threshold identification refers to the process of identifying the stimulus level at which a user can correctly detect the stimulus a predefined percentage of the time, e.g. 75\%. The result from such detection task is conventionally modeled as a psychometric function, which describes the relationship between the stimulus level (x-axis) and the chance of a certain response (y-axis). Depending on the question posed to the user, there are two types of psychometric functions. In a yes/no task, the user is exposed to one stimulus per trial and is asked the question: "Did you detect the stimulus?". The $\mathrm{y}$-axis of the psychometric function in this case represents the percentage of "yes" responses, ranging from 0 at zero stimulus level to 1 at significantly high stimulus level. In a classical two-alternative forced-choice task (2AFC), the user is presented two options concurrently or sequentially in 2 intervals, only one of which contains the stimulus, and answers to the question: "In which interval was the stimulus present?". In this case, the y-axis of the psychometric function represents the percentage of correct responses, and ranges from 0.5 at zero stimulus level to 1 at significantly high stimulus level. Existing RDTs studies employed a variant of the classical 2AFC method where alternative questions such as: "Did you walk to the left or the right?" were presented. The psychometric curve derived from this variant is similar to the one from a yes/no task, where the y-axis ranges from 0 to 1 . Generally, the 2AFC method is preferred over the yes/no method due to the fact that the yes/no method often contains response bias, e.g. a tendency to answer "yes" when the stimulus is not perceivable. Therefore, originally in our pilot study, we employed the same $2 \mathrm{AFC}$ variant task as other existing RDTs studies for our experiment. However, one pilot subject consistently experienced the sensation of going left when the gains in both directions are sufficiently small, and thus keeping answering "left". This resulted in a right curvature radius for his left threshold. From an application point of view, this subject can never be redirected left because his left threshold is a right curve. When the same pilot subject was tested again with the classical 2AFC task, the left threshold were found to be a $\sim 13 \mathrm{~m}$ radius left curve. This finding suggests that the $2 \mathrm{AFC}$ variant task is not robust against this type of bias. As a result, for the identification of our curvature RDTs in this experiment, we use the classical 2AFC task.
Independent of the task used, the threshold identification process revolves around identifying the psychometric function. There are two main types of methods used for threshold identification. The first type, the constant stimuli method (CSM), fits the whole psychometric function based on users' responses at a pre-selected range of stimuli. In order to obtain a well-fitted curve, many stimulus levels are required and a high number of repetitions is usually needed at each tested stimulus level. For a threshold identification application, this method may not be very efficient since not the whole curve, but only a certain point on the curve or the slope of the curve is normally of interest. The second type, the adaptive method, tackles the efficiency problem of the CSM by using an adaptive procedure which selects the next stimulus level based on the previous response(s) of the users. This type of method does not identify the whole psychometric function, but only some of its parameters such as threshold, slope, guessing rate, etc. One category of adaptive methods is called the maximum likelihood procedure in which the optimal placement of the next stimulus is determined by fitting a parametric model of the psychometric function using data collected from all previous trials. Depending on stimulus placement strategies, stopping conditions and threshold final estimation methods, there is a wide variety of maximum likelihood procedures, of which the Bayesian method is recommended when only the threshold needs to be identified [Treutwein 1995]. While the CSM is simpler to implement, more trials are required leading to longer exposure time of the subjects in the VE. Therefore, in our experiment, we adopt a Bayesian method called QUEST [Watson and Pelli 1983].

\subsection{Speed regulation}

In an experiment by Neth et al., walking speed has been shown to have a negative correlation with curvature RDTs [Neth et al. 2011]. In that experiment, walking speed was controlled by the subjects following and maintaining a distance to an object moving at constant speed. However, it is not known how such task distracts the users visually from the main task of detecting curvature redirection, and consequently how it affects RDTs. Therefore, in our experiment we control the users' speeds by having them following certain audio step rhythms. Dean studied the energy expenditure of human walking and derived a relationship between walking speed, stepping frequency and height [Dean 1965]. The equation describing this relationship is used for generating the aforementioned step rhythms.

\section{USER STUDY}

\subsection{Experiment Design}

The experiment scene contains an empty room with four surrounding walls and a red target located $7.5 \mathrm{~m}$ from the starting position of the user. Before the experiment, users' heights are collected and their personal step rhythms are generated.Each user has to walk with two speed conditions: $0.75 \mathrm{~m} / \mathrm{s}$ and $1.25 \mathrm{~m} / \mathrm{s}$ (similar to the conditions in [Neth et al. 2011]) regulated by the step rhythms. We also identify the left and right thresholds separately for each user, resulting in four threshold values of four psychometric functions to be found per user. Speed and curvature direction are therefore within-subject variables. 
Using the 2AFC method, in each trial each user walks to the red target two times. In only one of the two walks, a curvature gain is applied such that the subject walks on a curve in real life while seeing that he/she walks straight in the VE. The curvature gain is defined as the inverse of the radius of the curve that the subject walks on. The order is randomized between trials. Four separate "QUESTs" handle the four psychometric functions and calculate the next curvature gain value to be tested for each function. These four next values are selected randomly to be presented in the next trial. This process is called interleaving, commonly used to reduce training and adaption effect. For each "QUEST", 40 trials are required, resulting in a total of 160 trials per user. During the trials, the process of updating the "QUESTs" with users' responses and computing the next curvature gain value is automated. Users respond to the $2 \mathrm{AFC}$ question using a built-in eye tracker.

\subsection{Experiment Setup}

The experiment setup consists of an Oculus DK2 with a built-in SMI eye tracker and an Intersense IS-1200 inside-out optical tracking system mounted on top, providing 6-DOF positional tracking at $180 \mathrm{~Hz}$. A cover is added in front of the headset to prevent users from seeing the floor. The scenes are optimally designed in Unity to run at the HMD's maximum frame rate $75 \mathrm{~Hz}$. The whole setup is powered by a backpack-mounted notebook. The available tracking space is $12 \mathrm{~m} \mathrm{x} 6 \mathrm{~m}$. The users also wear noise cancelling headphones, where the step regulating rhythms are played.

\subsection{Participants and Procedure}

61 subjects (aged from 18-35 $(M=25.1, S D=3.9), 30$ male and 31 female, right-handed, with normal or corrected-to-normal vision, no vestibular dysfunction or injured) were recruited through the university market place. The experiment lasted about 90 minutes including instruction and set up time and was divided into two sessions. The subjects were paid $15 \mathrm{CHF} /$ hour for their contribution. In the first session, the subjects were informed about the purpose of the experiment and signed the consent form to participate. Subjects provided basic information such as age, handedness, and gaming hours per week. They also were informed about the risk of motion sickness and filled out Kennedy's simulator sickness questionnaire (SSQ) [Kennedy et al. 1993]. The subjects were then shown different screenshots of the experiment scene and given the following instruction: "When the program starts, you will see a starting position. Walk to this position. A scene containing a red target will appear. Wait until you hear the step sound and then start walking in the same frequency as the sound towards the target. When you reach the target, a second starting position will appear. Do the same as before and you will see the red target again. Walk to the target again. After you reach the target, a question will appear on the screen asking you: In which walk were you redirected? Select your answer (first or second) by looking at it, and look at the confirm button to confirm." After the subjects confirmed that they understood the task, they were given some trial runs. In these trial runs, a curvature gain of 0.2 (corresponding to a $5 \mathrm{~m}$ radius curve) was used. This value was chosen based on existing research such that it will be perceivable for all subjects and consequently they would get how it feels when being redirected. These trials included both curvature directions and both speed conditions, so that the subjects could get accustomed to following the different step rhythms corresponding to the different speeds. After the subjects finished the trial runs they could start the experiment. The experiment was designed such that the subjects would never be close to a wall if they followed the instructions. Therefore, an experimenter is always there but there is no need for him/her to walk behind the subjects. The subjects could take a break any time they want, but on average the subjects took a break every 20 minutes in each session. After each session, the subjects filled out the SSQ again.

\section{RESULTS AND DISCUSSION}

Out of 61 subjects, one female subject reported motion sickness after 10 minutes and could not continue. 60 remaining subjects could complete the whole experiment. Two-sample t-tests were conducted to compare the SSQ scores before and after each session. In both sessions, there was a significant increase in score in the after condition. To investigate the effect of gender on this increase in SSQ score, we fitted a linear mixed model which includes gender as a fixed factor and subject as a random factor: ScoreIncrease $1+$ Gender $+(1 \mid$ Subject $)$. Results showed no significant effect of gender on the increase in SSQ score.

For each subject, four threshold values were obtained corresponding to the two speeds and two curvature directions: left slow, left fast, right slow and right fast. The distribution of the 240 obtained threshold values (albeit interdependency between data points as each subject contributes four data points) is shown in Figure 1, serving only as an overall picture. In general, there is a high variability in curvature gain thresholds, ranging from 0.0237 (equivalent to a radius of $42.2 \mathrm{~m}$ ) to 0.1994 (equivalent to a radius of $5.2 \mathrm{~m}$ ) and a median of 0.0976 (equivalent to a radius of $10.24 \mathrm{~m}$ ), interquartile range 0.07 to 0.1336 . To investigate the effect of gender, speed and curvature direction, we fitted a linear mixed model which includes gender, speed and curvature direction as fixed factors and subjects as a random factor. We also included gaming hours per week as a fixed factor to investigate whether gaming experience influences the ability to detect curvature redirection. The full mixedeffect model therefore becomes: Threshold $\sim 1+$ Gender + Speed + CurvatureDirection + GameHours + (1|Subject). Results show no significant effect of curvature direction on RDTs $(p=0.2)$, indicating that right-handed subjects tend perform equally well at detecting curvature redirection when the curve is towards either left or right direction. There is also no significant effect of the number of gaming hours on RDTs $(p=0.6)$, which means gamers do not have an advantage over non-gamers at detecting redirection. The significant effects on curvature RDTs are speed (Figure 2a) and gender (Figure $2 \mathrm{~b}$ ) with $p<0.001$ and $p=0.012$ respectively. In Figure 2, the threshold values were adjusted to isolate the effect of the independent variable of interest while the effects of all other independent variables are averaged. The negative slope in Figure 2a indicates the faster the walking speed, the lower the curvature RDTs, i.e. higher sensitivity. This result is in line with results in [Neth et al. 2011], although their method of regulating walking speed is different from ours. Finally, Figure $2 \mathrm{~b}$ shows that gender significantly affects curvature RDTs, and that male subjects tend to 


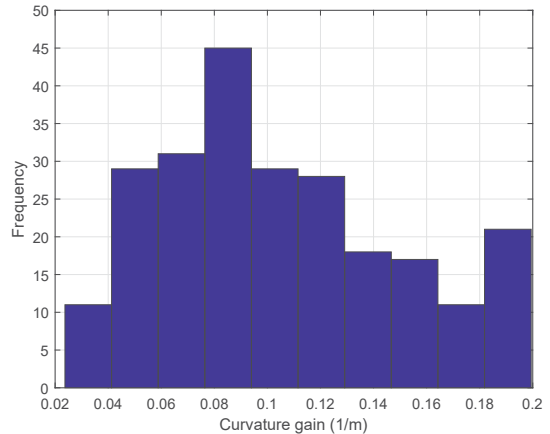

Figure 1: Distribution of all obtained threshold values

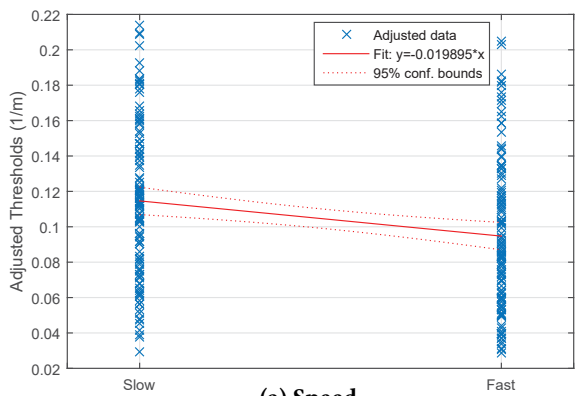

(a) Speed

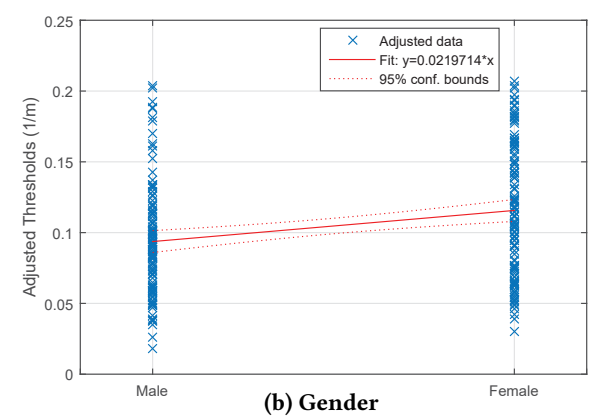

Figure 2: Effects of Speed and Gender on curvature RDTs

be more sensitive in detecting curvature gain. The average curvature radius threshold for men is $10.7 \mathrm{~m}$ while it is $8.63 \mathrm{~m}$ for women. This result also confirms the observed tendency found previously in [Bruder et al. 2009].

\section{CONCLUSION}

In this paper, we proposed a nonvisual method of regulating walking speed using audio step rhythms. We observed that there is a high variability in individuals' curvature gain thresholds. We found that right-handed subjects tend to be equally sensitive to either curve direction and gamers do not have an advantage in detecting redirection. We were able to reproduce existing results on the effect of walking speed on curvature RDTs. Finally, the obtained results supported our hypothesis about gender difference in detecting redirection and we found that men are more sensitive to curvature redirection than women.

The findings from this experiment indicate that curvature gains should be carefully selected for individuals and applied dynamically depending on their walking speed to maintain immersion.
Although gender is correlated with RDTs, there is quite high variance in RDTs within each gender. Therefore, a measure of individual visual dependence could potentially be a better predictor for RDTs. Nevertheless, this hypothesis remains to be investigated in future experiments.

\section{ACKNOWLEDGMENTS}

This work is funded by the Swiss National Science Foundation (Award Number: CR23I2_162752).

\section{REFERENCES}

M. Barnett-Cowan, R. T. Dyde, C. Thompson, and L. R. Harris. 2010. Multisensory determinants of orientation perception: task-specific sex differences. European fournal of Neuroscience 31, 10 (2010), 1899-1907. https://doi.org/10.1111/j.1460-9568. 2010.07199.x

Gerd Bruder, Frank Steinicke, Klaus H Hinrichs, Harald Frenz, and Markus Lappe. 2009. Impact of Gender on Discrimination between Real and Virtual Stimuli. In Workshop on Perceptual Illusions in Virtual Environments. Citeseer, 10-15.

Cynthia L Darlington and Paul F Smith. 1998. Further evidence for gender differences in circularvection. Journal of Vestibular Research 8, 2 (1998), 151-153.

G. A. Dean. 1965. An Analysis of the Energy Expenditure in Level and Grade Walking. Ergonomics 8, 1 (1965), 31-47. https://doi.org/10.1080/00140136508930772 arXiv:http://dx.doi.org/10.1080/00140136508930772

Timofey Grechkin, Jerald Thomas, Mahdi Azmandian, Mark Bolas, and Evan Suma. 2016. Revisiting Detection Thresholds for Redirected Walking: Combining Translation and Curvature Gains. In Proceedings of the ACM Symposium on Applied Perception (SAP '16). ACM, New York, NY, USA, 113-120. https://doi.org/10.1145/ 2931002.2931018

Eric Hodgson, Eric Bachmann, and Tyler Thrash. 2014. Performance of Redirected Walking Algorithms in a Constrained Virtual World. IEEE Transactions on Visualization and Computer Graphics 20, 4 (April 2014), 579-587. https://doi.org/10.1109/ TVCG.2014.34

Robert S. Kennedy, Lawrence J. Hettinger, Deborah L. Harm, Ordy J. Mark, and William P. Dunlap. 1996. Psychophysical scaling of circular vection (CV) produced by optokinetic $(\mathrm{OKN})$ motion: individual differences and effects of practice. Journal of Vestibular Research 6, 5 (1996), 331-341.

Robert S. Kennedy, Norman E. Lane, Kevin S. Berbaum, and Michael G. Lilienthal. 1993. Simulator Sickness Questionnaire: An Enhanced Method for Quantifying Simulator Sickness. The International fournal of Aviation Psychology 3, 3 (1993), 203-220. https://doi.org/10.1207/s15327108ijap0303 arXiv:http://dx.doi.org/10.1207/s15327108ijap0303

Christian T. Neth, Jan L. Souman, David Engel, Uwe Kloos, Heinrich H. Bülthoff, and Betty J. Mohler. 2011. Velocity-dependent dynamic curvature gain for redirected walking. In 2011 IEEE Virtual Reality Conference. IEEE, New York, NY, USA, 151-158. https://doi.org/10.1109/VR.2011.5759454

Anh Nguyen, Yannick Rothacher, Peter Brugger, Bigna Lenggenhager, and Andreas Kunz. 2016. Estimation of Individual Redirected Walking Thresholds Using Standard Perception Tests. In Proceedings of the 22nd ACM Conference on Virtual Reality Software and Technology (VRST '16). ACM, New York, NY, USA, 329-330. https: //doi.org/10.1145/2993369.2996304

Norbert Nitzsche, Uwe D. Hanebeck, and Günther Schmidt. 2004. Motion Compression for Telepresent Walking in Large Target Environments. Presence 13, 1 (Feb 2004), 44-60. https://doi.org/10.1162/105474604774048225

Sharif Razzaque, Zachariah Kohn, and Mary C. Whitton. 2001. Redirected Walking. In Eurographics 2001 - Short Presentations. Eurographics Association, Geneva, Switzerland, 1-6. https://doi.org/10.2312/egs.20011036

Frank Steinicke, Gerd Bruder, Jason Jerald, Harald Frenz, and Markus Lappe. 2010. Estimation of Detection Thresholds for Redirected Walking Techniques. IEEE Transactions on Visualization and Computer Graphics 16, 1 (Jan. 2010), 17-27. https: //doi.org/10.1109/TVCG.2009.62

Luc Tremblay, Digby Elliott, and Janet L Starkes. 2004. Gender Differences in Perception of Self-Orientation: Software or Hardware? Perception 33, 3 (2004), 329-337. https: //doi.org/10.1068/p5209 arXiv:https://doi.org/10.1068/p5209 PMID: 15176617.

Bernhard Treutwein. 1995. Adaptive Psychophysical Procedures. Vision Research 35, 17 (1995), 2503 - 2522. https://doi.org/10.1016/0042-6989(95)00016-X

Martin Usoh, Kevin Arthur, Mary C. Whitton, Rui Bastos, Anthony Steed, Mel Slater, and Frederick P. Brooks, Jr. 1999. Walking $>$ Walking-in-place $>$ Flying, in Virtual Environments. In Proceedings of the 26th Annual Conference on Computer Graphics and Interactive Techniques (SIGGRAPH '99). ACM Press/Addison-Wesley Publishing Co., New York, NY, USA, 359-364. https://doi.org/10.1145/311535.311589

Andrew B. Watson and Denis G. Pelli. 1983. Quest: A Bayesian adaptive psychometric method. Perception \& Psychophysics 33, 2 (01 Mar 1983), 113-120. https://doi.org/ 10.3758/BF03202828 